\section{Psychological Medicine}

cambridge.org/psm

\section{Correspondence}

Cite this article: Mitchell KS, Wolf EJ, Lyons MJ, Goldberg J, Magruder KM (2018). A co-twin control study of the association between combat exposure, PTSD and obesity in male veterans. Psychological Medicine 48, 2449-2452. https://doi.org/10.1017/ S0033291718001514

Received: 13 February 2018

Revised: 11 May 2018

Accepted: 15 May 2018

First published online: 18 June 2018

Author for correspondence: K.S. Mitchell, E-mail: Karen.Mitchell5@va.gov

\title{
A co-twin control study of the association between combat exposure, PTSD and obesity in male veterans
}

K. S. Mitchell1,2, E. J. Wolf ${ }^{1,2}$, M. J. Lyons ${ }^{3}$, J. Goldberg ${ }^{4,5}$ and K. M. Magruder 6,7

\footnotetext{
${ }^{1}$ National Center for PTSD, VA Boston Healthcare System, Boston, MA, USA; ${ }^{2}$ Department of Psychiatry, Boston University School of Medicine, Boston, MA, USA; ${ }^{3}$ Department of Psychological and Brain Sciences, Boston University, Boston, MA, USA; ${ }^{4}$ Seattle VA Epidemiology Research and Information Center, VA Puget Sound Health Care System, Seattle, WA, USA; ${ }^{5}$ Department of Epidemiology, University of Washington, Seattle, WA, USA; ${ }^{6}$ Department of Psychiatry and Behavioral Sciences, Medical University of South Carolina, Charleston, SC, USA and ${ }^{7}$ Department of Public Health Sciences, Medical University of South Carolina, Charleston, SC, USA
}

Trauma exposure has been associated with increased risk for obesity. Most studies have focused on childhood abuse. For example, a recent meta-analysis found that adults who reported childhood physical, sexual, or emotional abuse were more likely to be obese, and results did not differ by gender (Hemmingsson et al., 2014). However, adult onset trauma, including combat exposure, may also be associated with obesity and obesity-related illnesses (Boscarino, 1997; Crum-Cianflone et al., 2014).

Posttraumatic stress disorder (PTSD) also is associated with an increased risk for obesity, although this relationship may vary by gender: the PTSD-obesity relation has proved fairly robust among women but not men (Perkonigg et al., 2009; Mitchell et al., 2013). However, recent findings suggest that PTSD may be associated with obesity among men over the age of 60 , possibly because older adults may be more vulnerable to health conditions secondary to PTSD (Smith et al., 2015).

Prior work suggests that trauma exposure is associated with obesity via PTSD (Mitchell et al., 2013), but trauma may also be independently linked to obesity, given that chronic stress is associated with elevated cortisol levels and palatable food cravings (see Hemmingsson et al., 2014). We hypothesize that both trauma exposure and PTSD play a causal role in the development of obesity. PTSD and trauma have been associated with neuroendocrine dysregulation, unhealthy eating habits, and eating disorders, which may contribute to weight gain (Mitchell et al., 2012; Crum-Cianflone et al., 2014). Obesity, via increased inflammation, also may contribute to the development of PTSD in response to trauma. However, there is little evidence for this mechanism (Masodkar et al., 2016).

A third possibility is that, rather than trauma/PTSD contributing to the development of obesity or vice versa, genetic liability may predispose some individuals to trauma exposure, PTSD, and obesity (Sumner et al., 2017). Twin studies are one way to investigate these competing hypotheses. Case-control co-twin designs, in which one member of a twin pair possesses a trait, such as obesity, while the other twin does not, are a unique approach to investigating associations among phenotypes while controlling for genetic factors, childhood family environment, and age. Twins are selected based on discordance for the outcome, and the impact of within-pair differences in exposures are examined (Goldberg and Fischer, 2014). In this case, a significant association between two traits among discordant monozygotic (MZ) twins indicates that the relation is not explained by genes and implicates trauma/PTSD in the development of obesity. Significant associations among discordant dizygotic (DZ) twins but not MZs suggest that genes are at least partly responsible for their overlap (Goldberg and Fischer, 2014). Results of sibling studies can be biased if confounders are not perfectly shared among siblings. However, it is appropriate to use these designs when confounders are more similar among siblings than the exposure (Frisell et al., 2012). We tested our hypothesis that PTSD and trauma would be predictive of obesity in later life, as evidenced by significant trauma/PTSD_obesity associations in discordant male twin pairs from the Vietnam Era Twin Registry (VETR).

\section{Method}

\section{Participants and procedures}

VETR participants are male twins who were born between 1939 and 1957 and served in the US military between 1964 and 1975. Twins first completed a mailed survey in 1987. Lifetime PTSD diagnoses were first assessed via phone interview in 1992 (time 1; T1). Time 2 (T2) data collection for this study was by mailed survey and telephone interview in 2010-2012 (Magruder et al., 2016). Participants provided written informed consent; approvals were 
obtained from local institutional review boards. Body mass index (BMI) data were available for 4862 twins at T2; 137 twin pairs $(\mathrm{MZ}=64, \mathrm{DZ}=73)$ were discordant for obesity at $\mathrm{T} 2$. Of this group, $94.16 \%$ were White, $4.38 \%$ were Black, and $1.46 \%$ were Native American; 0.7\% were Hispanic/Latino.

\section{Measures}

\section{Lifetime PTSD diagnoses}

The Mental Health Diagnostic Interview Schedule, Version III-Revised (DIS-III-R; Robins et al., 1998) was administered via telephone in 1992 (T1). Inter-rater reliability for the PTSD diagnosis was $\kappa=0.54$. We also analyzed a continuous score of lifetime PTSD symptoms.

\section{Trauma exposure}

The Composite International Diagnostic Interview [CIDI; (Kessler and Ustün, 2004)] was used to assess trauma exposure. Analyses of these data were limited to the following interpersonal trauma types: childhood parental abuse, childhood abuse by another relative, rape, sexual assault other than rape, unexpected death of a loved one, and witnessing violence. This was done because interpersonal trauma and combat exposure have been associated with poorer outcomes compared with non-interpersonal traumas (e.g., natural disasters); unexpected death of a loved one was included as it is the most commonly endorsed trauma type in the general population (Kessler et al., 1995; Prigerson et al., 2001). Because rates of childhood parental abuse $(\mathrm{MZ}=4)$ and rape $(\mathrm{MZ}=4, \mathrm{DZ}=3)$ were quite low, we combined childhood parental and other relative abuse into an 'any childhood abuse' variable $(n=28)$, and we combined rape and other sexual assault other into an 'any sexual assault' variable $(n=19)$. We also created a binary 'any interpersonal trauma' variable.

We included the Combat Exposure Index, administered in the 1987 questionnaire, which assesses exposure to specific types of potentially traumatic combat experiences and is a valid and reliable indicator of combat exposure in the VETR (Janes et al., 1991). Responses were categorized $0=$ non-Southeast Asia service or Southeast Asia service without combat and $1=$ ANY combat trauma exposure.

\section{Obesity}

$\mathrm{BMI}$ at $\mathrm{T} 1$ and $\mathrm{T} 2$ was calculated from self-report height and weight. Obesity was defined as BMI $\geqq 30.0$ (World Health Organization, 2000). Discordance was defined as the BMI of one twin being $\geqq 30$, with the BMI of the other twin being $<30$, and at least a three-point difference in BMI between the co-twins.

\section{Covariates}

We included lifetime major depressive disorder (MDD), assessed using the DIS-III-R, and highest grade completed, as covariates.

\section{Zygosity}

Zygosity in the VETR was determined through the use of DNA, blood group, and questionnaire methods (Forsberg et al., 2010).

\section{Statistical analysis}

Analyses were conducted in SAS 9.4. We used tetrachoric correlations to compare sibling-pair sharing for the independent variables. Conditional logistic regression models, estimated via SAS PROC LOGISTIC with family ID as a stratification variable and obesity status as the outcome, were used to calculate matchedpairs odds ratios (OR) for discordant twins. All exposures were tested in separate models. We first estimated crude models and then added $\mathrm{T} 1$ obesity as a covariate in order to investigate the impact of exposures on obesity in later life. For independent variables significantly associated with obesity, we estimated a third model controlling for MDD and education as well as T1 obesity.

\section{Results}

\section{Descriptives}

MZ twins were more likely to participate at T2 (63.4\%) compared with DZ twins $(60.4 \% ; p=0.02)$. See Table 1 for rates of trauma exposure, lifetime PTSD, and mean PTSD symptom scores by zygosity and weight status. Obese twins had higher rates of combat exposure, and most other trauma exposures, compared with non-obese twins. Within-pair correlations for MDD $(r=0.30)$ and education $(r=0.61)$ were equal to or higher than correlations for the other independent variables $(r=0.19-0.30)$, with the exception of unexpected death $(r=0.37)$, childhood abuse $(r=$ $0.83)$, and sexual assault $(r=0.47)$, generally supporting the use of the co-twin design.

\section{Discordant twin analyses}

Lifetime PTSD diagnoses were significantly associated with obesity status among DZ twins only; however, this association became non-significant when controlling for $\mathrm{T} 1$ obesity (see Table 1). Continuous PTSD symptoms, childhood abuse, sexual assault, witnessing violence, and unexpected death of a loved one were not associated with obesity status. We further explored whether meeting criteria for PTSD symptom clusters (re-experiencing, avoidance, and hyperarousal) was associated with obesity; these results were non-significant as well.

$\mathrm{MZ}$ and DZ twin pair members who endorsed combat exposure in Southeast Asia were more likely to be obese as well. This association remained significant when controlling for T1 obesity and MDD and education ( $p=0.02$ and 0.005 , respectively) and when controlling for these variables as well as T1 lifetime PTSD diagnoses ( $p=0.03$ and 0.01 , respectively).

\section{Discussion}

Combat exposure was significantly associated with increased likelihood of obesity among MZ and DZ obesity-discordant twins, even after controlling for T1 obesity, MDD, PTSD, and education, suggesting a link between combat and development of obesity in later life. Combat exposure may have particularly deleterious consequences among samples of veterans that are enriched for trauma, compared with other forms of trauma (Prigerson et al., 2001). Although the exact mechanisms are unclear, stress can lead to neuroendocrine dysregulation, which can contribute to weight gain, or unhealthy eating habits may be used as a form of 'self-medication' (Crum-Cianflone et al., 2014). Our results underscore the importance of considering the impact of combat exposure on veterans' health throughout the life-course, especially given that participants were exposed to combat 37-48 years before assessment of obesity (Vieweg et al., 2007). Although twice as many obese DZ twins as non-obese DZ twins had lifetime PTSD diagnoses, the association between diagnosis and obesity was not significant after controlling for T1 obesity. This may 
Table 1. Matched-pairs odds ratios for the influence of exposures on obesity

\begin{tabular}{|c|c|c|c|c|c|c|c|c|c|c|c|}
\hline \multirow{3}{*}{\multicolumn{2}{|c|}{ Exposures }} & \multicolumn{5}{|c|}{$\begin{array}{l}\text { MZ twins } \\
n=64 \text { pairs }\end{array}$} & \multicolumn{5}{|c|}{$\begin{array}{l}\text { DZ twins } \\
n=73 \text { pairs }\end{array}$} \\
\hline & & \multirow{2}{*}{$\begin{array}{c}M Z_{\circ} \\
\text { No. (\%) }\end{array}$} & \multirow{2}{*}{$\begin{array}{c}M Z_{n-\mathrm{o}} \\
\text { No. (\%) }\end{array}$} & \multirow[b]{2}{*}{ B } & \multirow[b]{2}{*}{ S.E. } & \multirow[b]{2}{*}{ OR $(95 \% \mathrm{Cl})$} & \multirow{2}{*}{$\begin{array}{c}\mathrm{DZ} \\
\text { No. (\%) }\end{array}$} & \multirow{2}{*}{$\begin{array}{c}\mathrm{DZ}_{n-\mathrm{o}} \\
\text { No. (\%) }\end{array}$} & \multirow[b]{2}{*}{ B } & \multirow[b]{2}{*}{ S.E. } & \multirow[b]{2}{*}{ OR $(95 \% \mathrm{Cl})$} \\
\hline & & & & & & & & & & & \\
\hline \multicolumn{2}{|l|}{ PTSD diagnosis (T1) } & $5(7.81)$ & $8(12.50)$ & -0.47 & 0.57 & $0.63(0.20-1.91)$ & $12(16.44)$ & $2(2.74)$ & 1.79 & 0.76 & $6.00(1.34-26.81)$ \\
\hline \multicolumn{2}{|l|}{ PTSD diagnosis ( $\mathrm{T} 1)$, controlling for $\mathrm{T} 1$ obesity } & & & -0.34 & 0.59 & $0.71(0.23-2.25)$ & & & 1.50 & 0.78 & $4.50(0.97-20.83)$ \\
\hline \multicolumn{2}{|c|}{ Met criteria for PTSD re-experiencing symptom cluster ( $\mathrm{T} 1$ ), controlling for obesity } & $14(23.33)$ & $21(37.50)$ & -0.69 & 0.50 & $0.50(0.19-1.33)$ & $18(28.13)$ & $13(20.63)$ & -0.13 & 0.52 & $0.88(0.32-2.41)$ \\
\hline \multicolumn{2}{|c|}{ Met criteria for PTSD avoidance symptom cluster (T1), controlling for obesity } & $7(11.86)$ & $7(12.50)$ & 0.00 & 0.71 & $1.00(0.25-4.00)$ & $8(12.50)$ & $2(3.17)$ & 0.69 & 0.87 & $2.00(0.37-10.92)$ \\
\hline \multicolumn{2}{|c|}{ Met criteria for PTSD hyperarousal symptom cluster (T1), controlling for obesity } & $11(18.33)$ & $14(25.00)$ & -0.56 & 0.63 & $0.57(0.17-1.95)$ & $11(17.19)$ & $8(12.70)$ & 0.29 & 0.54 & $1.33(0.46-3.84)$ \\
\hline \multicolumn{2}{|l|}{ Any childhood abuse } & $4(6.25)$ & $5(7.81)$ & 0.69 & 1.22 & $2.00(0.18-22.06)$ & $9(12.33)$ & $9(12.33)$ & 0.69 & 1.22 & $2.00(0.18-22.06)$ \\
\hline \multicolumn{2}{|l|}{ Any childhood abuse, controlling for T1 obesity } & & & $<0.001$ & 1.41 & $1.00(0.63-15.99)$ & & & 0.69 & 1.22 & $2.00(0.18-22.06)$ \\
\hline \multicolumn{2}{|l|}{ Any sexual assault } & $8(12.50)$ & $4(6.25)$ & 0.92 & 0.84 & $2.50(0.49-12.89)$ & $4(5.48)$ & $3(4.11)$ & -0.41 & 0.91 & $0.67(0.11-3.99)$ \\
\hline \multicolumn{2}{|l|}{ Any sexual assault, controlling for T1 obesity } & & & 1.61 & 1.10 & $5.00(0.58-42.80)$ & & & 0.69 & 1.22 & $2.00(0.18-22.06)$ \\
\hline \multicolumn{2}{|l|}{ Death of a loved one } & $28(43.75)$ & $21(32.81)$ & -0.22 & 0.67 & $0.80(0.22-2.98)$ & $35(47.95)$ & $28(38.36)$ & -0.29 & 0.54 & $0.75(0.26-2.16)$ \\
\hline \multicolumn{2}{|l|}{ Death of a loved one, controlling for T1 obesity } & & & -0.29 & 0.76 & $0.75(0.17-3.35)$ & & & -0.15 & 0.56 & $0.86(0.29-2.55)$ \\
\hline \multicolumn{2}{|l|}{ Witnessed violence } & $23(35.94)$ & $25(39.06)$ & -0.20 & 0.45 & $0.82(0.34-1.97)$ & $30(41.40)$ & $21(28.77)$ & 0.85 & 0.49 & $2.33(0.90-6.07)$ \\
\hline \multicolumn{2}{|l|}{ Witnessed violence, controlling for T1 obesity } & & & -0.25 & 0.50 & $0.78(0.29-2.09)$ & & & 0.69 & 0.55 & $2.00(0.68-5.85)$ \\
\hline \multicolumn{2}{|l|}{ Any IPT } & $40(62.50)$ & $39(60.94)$ & -1.10 & 0.82 & $0.33(0.07-1.65)$ & $50(68.49)$ & $44(60.27)$ & -1.10 & 1.15 & $0.33(0.04-3.21)$ \\
\hline \multicolumn{2}{|l|}{ Any IPT, controlling for T1 obesity } & & & -1.61 & 1.10 & $0.20(0.02-1.71)$ & & & -1.10 & 1.15 & $0.33(0.04-3.21)$ \\
\hline \multicolumn{2}{|l|}{ Combat } & $17(26.56)$ & $5(7.81)$ & 1.87 & 0.76 & $6.50(1.47-28.80)$ & $25(34.25)$ & $6(8.22)$ & 2.25 & 0.74 & $9.50(2.21-40.79)$ \\
\hline \multicolumn{2}{|l|}{ Combat, controlling for $\mathrm{T} 1$ obesity } & & & 1.71 & 0.77 & $5.50(1.22-24.81)$ & & & 2.83 & 1.03 & $17.00(2.26-127.74)$ \\
\hline \multicolumn{2}{|c|}{$\begin{array}{l}\text { Combat, controlling for T1 obesity, major depressive disorder, and highest grade } \\
\text { completed }\end{array}$} & & & 1.88 & 0.82 & $6.55(1.32-32.43)$ & & & 3.68 & 1.31 & $39.55(3.05-512.319)$ \\
\hline $\begin{array}{l}\text { Combat, controlling for T1 obesity, major depressive disorder, hig } \\
\text { completed, and T1 PTSD diagnosis }\end{array}$ & grade & & & 1.93 & 0.87 & $6.87(1.25-37.72)$ & & & 3.22 & 1.29 & $25.04(1.99-315.40)$ \\
\hline Mean (s.D.) & Mean (s.o.) & B & S.E. & OR & $95 \% \mathrm{Cl})$ & Mean (s.o.) & Mea & $n($ (s.o.) & B & S.E & OR $(95 \% \mathrm{Cl})$ \\
\hline T1 PTSD symptoms ${ }^{a}$ & $2.31(3.82)$ & -0.02 & 0.05 & 0.98 & $(0.88-1$ & $3.36(4.92)$ & 1.62 & (2.99) & 0.12 & 0.05 & $1.13(1.02-1.24)$ \\
\hline T1 PTSD symptoms, ${ }^{a}$ controlling for T1 obesity & & -0.03 & 0.06 & 0.98 & $(0.88-1$ & .09) & & & 0.09 & 0.06 & $1.09(0.98-1.22)$ \\
\hline
\end{tabular}

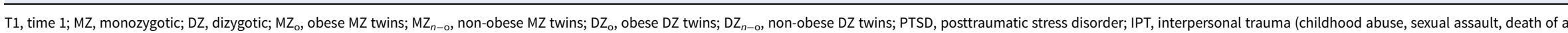

loved one, or witnessed violence); B, regression coefficient; s.E., standard error; OR, odds ratio; $\mathrm{Cl}$, confidence interval. Significant $(p<0.05)$ results are presented in bold-faced type.
aPTSD symptom scores were created by summing the number of PTSD criteria endorsed. 
have been due to our modest sample size of obesity-discordant pairs, given that previous studies of older male samples have found a positive PTSD-obesity relation (Smith et al., 2015).

These findings should be considered in light of several limitations. Results from this middle-aged, male, primarily white sample may not generalize to younger men, women, or other racial/ethnic groups. We were unable to rule out reverse causation, i.e., that obesity predisposes one to develop PTSD, although previous work suggests that PTSD is more likely to precede the onset of obesity than the reverse (Masodkar et al., 2016). We were unable to control for psychotropic medication use, which is a potential confounder. Zygosity was associated with T2 attrition, although it is unlikely that missing twin pairs differ systematically in terms of the combat-obesity associated observed in our data. Strengths of this study include the relatively large sample of twins and the discordant twin pair design. In sum, our findings are consistent with causal associations between combat exposure and obesity. Future studies should more thoroughly investigate longitudinal associations among these variables, eating behaviors among veterans with a history of combat exposure, and possible biological pathways for intervention among veterans with combat trauma.

Acknowledgements. The authors gratefully acknowledge the continued cooperation and participation of the members of the VET-Registry: without their contribution, this research would not have been possible. The contents of this article do not represent the views of the US Department of Veterans Affairs or the US Government.

Financial support. The Cooperative Studies Program (CSP) of the US Department of Veterans Affairs' Office of Research and Development has provided financial support for Cooperative Study \#569 and the development and maintenance of the Vietnam-Era Twin (VET) Registry. This work additionally was supported by a Career Development Award from the US Department of Veterans Affairs, Clinical Sciences Research and Development Program (EJW), by a Presidential Early Career Award for Scientists and Engineers (EJW) and administered by US Department of Veterans Affairs (VA) Office of Research and Development (PECASE 2013A), and by an award from the National Institutes of Health/National Institute on Aging (R01 AG018384 to MJL).

Conflicts of interest. None.

Ethical standards. The authors assert that all procedures contributing to this work comply with the ethical standards of the relevant national and institutional committees on human experimentation and with the Helsinki Declaration of 1975, as revised in 2008.

\section{References}

Boscarino JA (1997) Diseases among men 20 years after exposure to severe stress: implications for clinical research and medical care. Psychosomatic Medicine 59, 605-614.

Crum-Cianflone NF, Bagnell ME, Schaller E, Boyko EJ, Smith B, Maynard C, Ulmer CS, Vernalis M and Smith TC (2014) Impact of combat deployment and posttraumatic stress disorder on newly reported coronary heart disease among US active duty and reserve forces. Circulation 129, 1813-1820.

Forsberg CW, Goldberg J, Sporleder J and Smith NL (2010) Determining zygosity in the Vietnam era twin registry: an update. Twin Research and
Human Genetics: The Official Journal of the International Society for Twin Studies 13, 461-464.

Frisell T, Öberg S, Kuja-Halkola R and Sjölander A (2012) Sibling comparison designs: bias from non-shared confounders and measurement error. Epidemiology (Cambridge, Mass.) 23, 713-720.

Goldberg $\mathbf{J}$ and Fischer $\mathbf{M}$ (2005) Co-twin control methods. In Everitt B and Howell D (eds) Encyclopedia of Statistics in Behavioral Science. Hoboken, NJ: John Wiley \& Sons, Ltd.

Hemmingsson E, Johansson K and Reynisdottir S (2014) Effects of childhood abuse on adult obesity: a systematic review and meta-analysis. Obesity Reviews: An Official Journal of the International Association for the Study of Obesity 15, 882-893.

Janes GR, Goldberg J, Eisen SA and True WR (1991) Reliability and validity of a combat exposure index for Vietnam era veterans. Journal of Clinical Psychology 47, 80-86.

Kessler RC, Sonnega A, Bromet E, Hughes M and Nelson CB (1995) Posttraumatic stress disorder in the National Comorbidity Survey. Archives of General Psychiatry 52, 1048-1060.

Kessler RC and Ustün T (2004) The world mental health (WMH) survey initiative version of the world health organization (WHO) composite international diagnostic interview (CIDI). International Journal of Methods in Psychiatric Research 13, 93.

Magruder KM, Goldberg J, Forsberg CW, Friedman MJ, Litz BT, Vaccarino V, Heagerty PJ, Gleason TC, Huang GD and Smith NL (2016) Long-term trajectories of PTSD in Vietnam-era veterans: the course and consequences of PTSD in twins. Journal of Traumatic Stress 29, 5-16.

Masodkar K, Johnson J and Peterson MJ (2016) A review of posttraumatic stress disorder and obesity: exploring the link. The Primary Care Companion for CNS Disorders 18. doi: 10.4088/PCC.15r01848.

Mitchell KS, Aiello AE, Galea S, Uddin M, Wildman D and Koenen KC (2013) PTSD and obesity in the Detroit neighborhood health study. General Hospital Psychiatry 35, 671-673.

Mitchell KS, Mazzeo SE, Schlesinger M, Brewerton T and Smith BN (2012) Comorbidity of partial and subthreshold PTSD among men and women with eating disorders in the National Comorbidity Survey-Replication study. International Journal of Eating Disorders 45, 307-315.

Perkonigg A, Owashi T, Stein MB, Kirschbaum C and Wittchen H-U (2009) Posttraumatic stress disorder and obesity: evidence for a risk association. American Journal of Preventive Medicine 36, 1-8.

Prigerson HG, Maciejewski PK and Rosenheck RA (2001) Combat trauma: trauma with highest risk of delayed onset and unresolved posttraumatic stress disorder symptoms, unemployment, and abuse among men. The Journal of Nervous and Mental Disease 189, 99-108.

Robins L, Helzer J, Cottler L and Golding E (1998) National Institute of Mental Health Diagnostic Interview Schedule, Version III-Revised. St. Louis, MO: Department of Psychiatry, Washington University in St. Louis.

Smith BN, Tyzik AL, Neylan TC and Cohen BE (2015) PTSD and obesity in younger and older veterans: results from the mind your heart study. Psychiatry Research 229, 895-900.

Sumner JA, Duncan LE, Wolf EJ, Amstadter AB, Baker DG, Beckham JC, Gelaye B, Hemmings S, Kimbrel NA, Logue MW, Michopoulos V, Mitchell KS, Nievergelt C, Rothbaum A, Seedat S, Shinozaki G and Vermetten E (2017) Letter to the editor: posttraumatic stress disorder has genetic overlap with cardiometabolic traits. Psychological Medicine 47, 2036-2039.

Vieweg WV, Julius DA, Bates J, Quinn JF, Fernandez A, Hasnain M and Pandurangi AK (2007) Posttraumatic stress disorder as a risk factor for obesity among male military veterans. Acta Psychiatrica Scandinavica 116, 483-487.

World Health Organization (2000) Obesity: Preventing and Managing the Global Epidemic. Geneva, Switzerland: WHO. 